\title{
The reduction of quantum invariants of 4-thickenings
}

\author{
by \\ Ivelina Bobtcheva (Ancona) and Frank Quinn (Blacksburg, VA)
}

\begin{abstract}
We study the sensibility of an invariant of 2-dimensional $\mathrm{CW}$ complexes in the case when it comes as a reduction (through a change of ring) of a modular invariant of 4-dimensional thickenings of such complexes: it is shown that if the Euler characteristic of the 2-complex is greater than or equal to 1 , its invariant depends only on homology. To see what is happening when the Euler characteristic is smaller than 1, we use ideas of Kerler and construct, from any tortile category, an invariant of 4-thickenings which, if the category is modular, is a normalization of the Reshetikhin-Turaev invariant, and if the category is symmetric, depends only on the spine and the second Whitney number of the thickening. Then we show that the so(3) quantum invariant at a 5 th root of unity has its reduction, and this reduction is able to distinguish complexes with the same homology groups when the Euler characteristic is smaller than 1.
\end{abstract}

\section{Introduction}

1.1. The work of Kerler [10] and Turaev [21] has shown that it is natural to look at the quantum invariants not just as invariants of 3-manifolds, but rather as invariants of 4-dimensional 2-handlebodies bounded by 3manifolds, or in other words, 4-thickenings of 2-complexes. The question we study here: how sensitive is an invariant of 2-complexes which has a "lift", i.e. can be obtained through a change of ring from an invariant of 4dimensional thickenings, was inspired by the long standing Andrews-Curtis conjecture in the topology of 2-complexes.

The (generalized) Andrews-Curtis conjecture [1] asserts that any simple homotopy equivalence between two 2-complexes $P$ and $P^{\prime}$ can be obtained through a 2-deformation, which consists in changing the attaching maps of the 2-cells by homotopy and expansions and collapses of disks of dimension at most two $\left({ }^{1}\right)$. A 2-deformation transforming $P$ into $P^{\prime}$ will be denoted by $\sigma: P \rightsquigarrow P^{\prime}$ and it induces an isomorphism on homology (resp. coho-

2000 Mathematics Subject Classification: Primary 57M20, 57M27; Secondary 57R56.

$\left({ }^{1}\right)$ In part of the literature, for example [8], this is actually called a 3-deformation, since it can be achieved through expansions and collapses of disks of dimension at most three. 
mology) with $\mathcal{R}$-coefficients denoted by $\sigma_{*}: H_{*}(P, \mathcal{R}) \rightarrow H_{*}\left(P^{\prime}, \mathcal{R}\right)$ (resp. $\left.\sigma^{*}: H^{*}\left(P^{\prime}, \mathcal{R}\right) \rightarrow H^{*}(P, \mathcal{R})\right)$. The AC-conjecture is expected to be false and different proposals for counterexamples have been made, but there seem to be a lack of tools for actually detecting them as such. An extensive reference on this problem is [8].

To any 2-dimensional CW complex $P$, there corresponds a presentation $\widehat{P}=\left\langle x_{1}, \ldots, x_{n} \mid R_{1}, \ldots, R_{m}\right\rangle$ of its fundamental group, determined by a choice of a spanning tree $T$ in the one-skeleton of the complex and orientation of the 1-cells not in $T$. Theorem 2.4 in [8] shows that the correspondence $P \mapsto \widehat{P}$ induces a bijection between the 2-deformation types of connected 2-dimensional $\mathrm{CW}$ complexes and the equivalence classes of finite presentations under the following moves:

(i) the places of $R_{1}$ and $R_{s}$ are interchanged;

(ii) $R_{1}$ is replaced with $g R_{1} g^{-1}$, where $g$ is any element in the group, or the reverse of such a move;

(iii) $R_{1}$ is replaced with $R_{1}^{-1}$;

(iv) $R_{1}$ is replaced with $R_{1} R_{2}$;

(v) adding an additional generator $y$ and an additional relator $y R$, where $R$ is any word in the $x_{i}$ 's, or the reverse of such a move.

We will refer to these six operations as $A C$-moves, and a sequence $\sigma$ of ACmoves which transforms $\widehat{P}$ into $\widehat{P}^{\prime}$ will be denoted by $\sigma: \widehat{P} \rightsquigarrow \widehat{P}^{\prime}$ and called a 2-deformation of $\widehat{P}$. We will compose 2-deformations on the left. The inverse $\widehat{P} \mapsto P$ of the bijection above is obtained by taking the one-point union of $n$ circles and attaching to them $m$ 2-cells as described by the relations. We will denote by $[P]$ (resp. $[\widehat{P}])$ the 2-equivalence class of $P$ (resp. $\widehat{P}$ ).

A decorated 2-complex $(P, w)$ is a 2-dimensional complex $P$ and a choice of a cohomology class $w \in H^{2}(P, \mathbb{Z} / 2)$. Then a 2-deformation $\sigma:(P, w) \rightsquigarrow$ $\left(P^{\prime}, w^{\prime}\right)$ of decorated complexes is given by a 2-deformation $\sigma: P \rightsquigarrow P^{\prime}$ such that $\sigma^{*}\left(w^{\prime}\right)=w$. The equivalence class of $(P, w)$ under these deformations will be denoted by $[P, w]$.

1.2. What makes the AC-phenomenon difficult to detect is that it disappears after taking one-point union with spheres. In fact, if two 2-complexes $X$ and $Y$ are simple homotopy equivalent, then for some $k$ there exists a 2-deformation $X \vee \bigvee^{k} S^{2} \rightsquigarrow Y \vee \bigvee^{k} S^{2}$ (see (40) in [8]). Therefore, an invariant $\mathcal{Z}_{\mathcal{K}}^{2}$ of 2 -complexes, which takes values in a commutative ring $\mathcal{K}$, may have some chance of detecting a counterexample to the AC-conjecture if the following two conditions are satisfied:

- it is multiplicative with respect to one-point union, i.e. $\mathcal{Z}_{\mathcal{K}}^{2}(X \vee Y)=$ $\mathcal{Z}_{\mathcal{K}}^{2}(X) \mathcal{Z}_{\mathcal{K}}^{2}(Y)$

- $\mathcal{Z}_{\mathcal{K}}^{2}\left(S^{2}\right)$ is zero or a zero divisor in $\mathcal{K}$. 
A $\mathbb{Z} / p$-valued invariant, called $Q_{\mathbb{Z} / p}$, with this property was introduced in [18] and studied in [3]. Its input is a finite semisimple symmetric monoidal category, which is taken to be one of the families described by GelfandKazhdan in [6], obtained as subquotients of $\bmod p$ representations of simple Lie algebras. An extensive numerical study of $Q_{\mathbb{Z} / p}$, described in [23], showed that all numerically generated examples come from a representation of the free group on the generators into a subgroup of $\mathrm{GL}_{N}(\mathbb{Z} / p)$ for some $N$, and in this representation every word has order $p$. Consequently, it was shown in [16] that any invariant possessing this property is not sensitive to the AC-phenomenon. Such a property is obviously very difficult to check and this criterion has a limited use for invariants taking values in finite fields. The present work offers another criterion concerning the class of invariants of 2-complexes which are reductions of invariants of 4-thickenings of such complexes. Surprisingly it is easy to see that many of the known invariants of 2-complexes are such reductions: all (except maybe one) numerically generated examples of $Q_{\mathbb{Z} / p}$, but also many of the HKR-type invariants constructed in [4] from triangular non-cosemisimple algebras.

1.3. A 4-thickening $M$ of a 2 -dimensional $\mathrm{CW}$ complex $P$, denoted by $(M, P)$, is an orientable 4-dimensional manifold together with a decomposition as a handlebody with $0-, 1$ - and 2-handles and an identification (as CW complexes) of the spine of the handlebody structure with $P$ through an embedding $\iota_{M, P}: P \rightarrow M$. In particular, $\iota_{M, P}$ induces isomorphism on homology. We will restrict ourselves to 4 -thickenings with a single 0 -handle. A 2-deformation of such 4-thickenings is given by a sequence of the following handle moves:

(a) creation or cancellation of a canceling 1-2 handle pair $\left({ }^{2}\right)$;

(b) changing the attaching maps of the 1- and 2-handles by isotopy.

Observe that these moves induce a 2-deformation on the spine. Moreover we have a well defined functor from the category of 4 -thickenings/2-deformations into the category of decorated 2-complexes/2-deformations given by $(M, P) \rightarrow\left(P, \iota_{M, P}^{*}\left(w_{2}(M)\right)\right)$, where $w_{2}(M)$ is the second Whitney class of $M$.

The word 4-thickening is supposed to stress not only the fact that a spine has been fixed, but also that we have weaker equivalence relations on the objects than diffeomorphisms: while changing the attaching map of a 2-handle by isotopy is equivalent to the creation and cancellation of 2-3 handle pairs, isotoping the attaching map of a 3-handle is not (known to

$\left({ }^{2}\right)$ A pair of an $(n+1)$-handle and an $n$-handle is called a canceling pair if the attaching sphere of the $(n+1)$-handle intersects the belt sphere of the $n$-handle in a single point. 
be) a 2-deformation. In this sense there will be some abuse of notation. For example we will use the notation $\pm \mathbb{C} P^{2}$ to denote the 4-thickenings of the sphere (not the smooth 4-manifolds) obtained by attaching a 2-handle of framing +1 (resp. -1 ) to $B^{4}$.

1.4. Let $\mathcal{R}, \mathcal{K}$ be commutative rings and $\varphi: \mathcal{K} \rightarrow \mathcal{R}$ be a ring homomorphism. Let also $\mathcal{Z}_{\mathcal{K}}^{4}$ be a $\mathcal{K}$-valued invariant of 4 -thickenings, satisfying the following conditions:

- $\mathcal{Z}_{\mathcal{K}}^{4}$ is multiplicative with respect to (boundary) connected sum, i.e. $\mathcal{Z}_{\mathcal{K}}^{4}\left(M_{1} \#_{\partial} M_{2}\right)=\mathcal{Z}_{\mathcal{K}}^{4}\left(M_{1}\right) \mathcal{Z}_{\mathcal{K}}^{4}\left(M_{2}\right)$

- $\mathcal{Z}_{\mathcal{R}}^{2}=\mathcal{Z}_{\mathcal{K}}^{4} \otimes_{\varphi} \mathcal{R}$ depends only on the spine of the 4-thickening.

Then $\mathcal{Z}_{\mathcal{R}}^{2}$ is multiplicative with respect to one-point union of 2-complexes, and we will call $\mathcal{Z}_{\mathcal{R}}^{2}$ an $\mathcal{R}$-reduction of $\mathcal{Z}_{\mathcal{K}}^{4}$. Suppose also that $\mathcal{Z}_{\mathcal{K}}^{4}$ can be normalized to a $\mathcal{K}^{\prime}$-valued invariant $\mathcal{Z}_{\mathcal{K}^{\prime}}^{\partial}$ of the boundary for some field extension $\mathcal{K}^{\prime}$ of $\mathcal{K}$. Under these assumptions, Theorem 2.11 states that when the Euler characteristic of $P$ is greater than or equal to $1, \mathcal{Z}_{\mathcal{R}}^{2}(P)$ depends only on the homology of $P\left({ }^{3}\right)$. The precise definitions and the proof are given in Section 2.

Section 3 puts together the results of [10] and [2] to show that any finite semisimple tortile category $\mathcal{C}$ over $\mathcal{K}$ leads to an invariant $\mathcal{Z}_{\mathcal{K}}^{4}$ of 4 thickenings under 2-deformations. Actually in [10] Kerler defines and proves the invariance of $\mathcal{Z}_{\mathcal{K}}^{4}$ when the category is modular, and it is shown in [2] that the modularity condition is not necessary to have invariance under 2-handle slides. In the modular case $\mathcal{Z}_{\mathcal{K}}^{4}$ can be normalized to the Reshetikhin-Turaev invariant of the boundary. If instead the category is symmetric, we show that $\mathcal{Z}_{\mathcal{K}}^{4}$ depends only on the spine and the second Whitney class of the 4 -thickening, and if in addition the twist morphisms in $\mathcal{C}$ are the identities, $\mathcal{Z}_{\mathcal{K}}^{4}$ depends only on the spine, i.e. defines an invariant of 2-complexes.

In Section 4 we study the reduction in the case of so(3)-type tortile categories defined over the ring $\mathcal{K}_{p}=\mathbb{Z}[v]$ where $v$ is a $p$ th root of unity. The smallest example of such a category with $p=5$ is explicitly presented in Section 5. In this case, $\mathcal{Z}_{\mathcal{K}_{p}}^{4}$ is a normalization of the Kirby-Melvin so(3)invariant [13]. Moreover, through the ring homomorphism $\varphi_{p}: \mathcal{K}_{p} \rightarrow \mathbb{Z} / p$, sending $v$ to 1 , we obtain $Q_{\mathbb{Z} / p}$ as a $\mathbb{Z} / p$-reduction of $\mathcal{Z}_{\mathcal{K}_{p}}^{4}$. The example is used to show that the reduction is able to distinguish complexes with the same homology groups when the Euler characteristic is smaller than 1. The discussion also relates the pth cyclotomic order of a 3-manifold, defined in [5], to the minimal Euler characteristic of a 4-manifold which it bounds.

$\left(^{3}\right)$ All suggestions for counterexamples of the AC-conjecture are in Euler characteristic 1 . 


\section{Reduction of modular invariants to the spine}

2.1. Define a Kirby link (K-link) to be a framed link in $S^{3}$ where some of the unknotted components of framing 0 have been dotted. We will assume that the dotted components bound disjoint Seifert surfaces which have only transverse intersections with the undotted components. Then a based oriented Kirby link (BOK-link) is a K-link where one has a fixed numbering, base points and orientation of the undotted components and a numbering and a set of disjoint oriented Seifert surfaces for the dotted components. A BOK-link which has $L$ as the underlying K-link will be denoted by $\widehat{L}$.

Any K-link $L$ represents a 4 -dimensional 2-handlebody $M_{L}$ (see [12]), where the attaching maps of the 1-handles are described by the dotted components (we are actually drawing the canceling 2-handles) and the attaching maps of the 2-handles are described by the undotted ones. If $L$ has $n$ dotted and $m$ undotted components, any $\widehat{L}$ defines a unique presentation $\widehat{P}_{L}=\left\langle x_{1}, \ldots, x_{n} \mid R_{1}, \ldots, R_{m}\right\rangle$ of $\pi_{1}\left(M_{L}\right)$, where $R_{i}=R_{i}\left(x_{1}, \ldots, x_{n}\right)$ is a (non-freely reduced) word in the $x_{j}$ 's and shows in which order and with which sign the $i$ th undotted component intersects the Seifert surfaces of the dotted ones, starting from the base point. Observe that a different choice of orientation, numbering and base points changes the representation by a 2-deformation, i.e. there is a well defined map $L \mapsto\left[\widehat{P}_{L}\right]$ from the set of $\mathrm{K}$-links onto the set of 2-equivalence classes of presentations $\left({ }^{4}\right)$. An example of a BOK-link is shown in Figure 1.

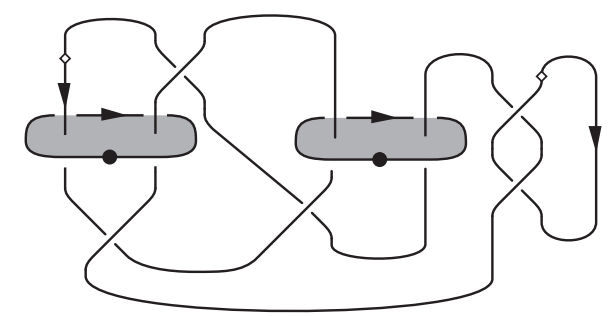

Fig. 1. A BOK-link $\widehat{L}$ with $\widehat{P}_{L}=\left\langle x, y \mid x y^{-1} x y, 1\right\rangle$

2.2. K- and BOK-links will be presented by plane tangle diagrams with blackboard framing. We recall that the framed tangles in $\mathbb{R}^{2} \times[0,1]$ form a category whose objects are sets of points in $\mathbb{R}^{2}$ and the morphisms are "slices" of framed links. The composition of two tangles is obtained by iden-

$\left({ }^{4}\right)$ The notion of K-link is equivalent to Kerler's notion of a bridged link introduced in [10] where the attaching maps of the 1-handles are described directly by two balls in $S^{3}$. But since bridged links are used to describe the boundary of the 4-thickening, the moves of equivalence there are stronger than the 2-deformation moves, including also the moves in 2.5 . 
(a)

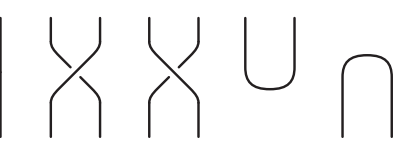

(b)

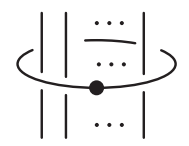

Fig. 2. Elementary tangle plane diagrams

tifying the outgoing ends of the first tangle with the incoming ends of the second. There is a product structure in this category given by disjoint union. The tangles are presented by their regular plane projections which consist of compositions and products of the elementary tangle diagrams presented in Figure 2(a). Note that we consider as incoming ends the bottom ones. The category of K-tangles is defined by introducing an additional elementary morphism corresponding to the diagram of Figure 2(b). Two K-links are (regular) isotopic if and only if the corresponding diagrams can be transformed into each other by the usual Reidemeister moves involving only undotted components and the additional moves presented in Figure 3.

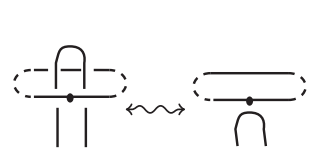

(a)

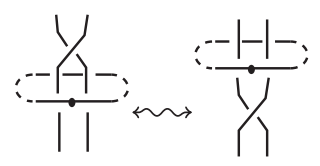

(b)

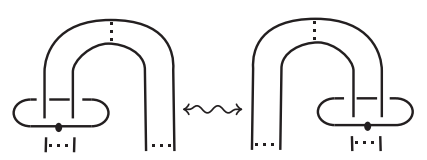

(c)

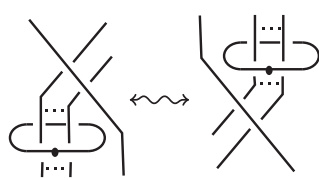

(d)

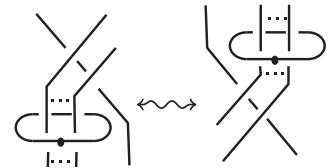

(e)

Fig. 3. Additional isotopy moves

2.5. Two K-links are said to be 2-equivalent if they can be deformed into each other through a sequence of the following moves (corresponding to 1- and 2-handle moves of the underlying handlebody), and changing a $\mathrm{K}$-link through such a sequence will be called a 2-deformation of this link:

(a) isotopy of framed links;

(b) any pair of one dotted component $x$ and one undotted component $y$ can be removed or added if the geometric intersection number of $y$ and a Seifert surface $S_{x}$ of $x$ is \pm 1 , while $S_{x}$ is disjoint from all other undotted components (1-2 handle cancellation or introduction);

(c) band-connected sum or difference of two undotted link components (sliding a 2-handle over a 2-handle);

2-deformation and 2-equivalence of BOK-links are defined analogously by 
adding the possibility of changing numberings, base points, Seifert surfaces and orientations.

We will denote by $L \rightsquigarrow L^{\prime}$ (resp. $\widehat{L} \rightsquigarrow \widehat{L}^{\prime}$ ) 2-deformations of K-links (resp. BOK-links) and we will compose such deformations on the left. By definition the K-links $L$ and $L^{\prime}$ are 2-equivalent if $M_{L}$ is 2-equivalent to $M_{L^{\prime}}$. Moreover, if $\tilde{\sigma}: \widehat{L}_{L} \rightsquigarrow \widehat{L}^{\prime}$ is a 2-deformation then $\widehat{P}_{L^{\prime}}$ is obtained from $\widehat{P}_{L}$ by one of the moves in 1.1. Therefore there is a well defined 2-deformation $\sigma$ which makes the following diagram commute:

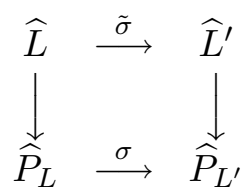

In a diagram like the one above we will call $\tilde{\sigma}$ the lift of $\sigma$ to $L$.

2.6. Not every 2-deformation of the spine can be lifted to a 2-deformation of its thickening. Let $\widehat{L}$ be a BOK-link. Then Proposition 5.5 in [4] states that if $\widehat{P}_{L}$ and $\widehat{P}^{\prime}$ are 2-equivalent, there exist 2-deformations $\tilde{\sigma}_{0}$ and $\xi$ such that the diagram

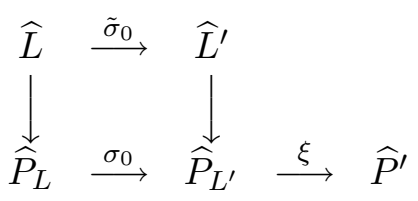

commutes, where $\xi$ is a sequence of cancellations of terms $x_{i} x_{i}^{-1}$ in the relations (considered as cyclic words in $x_{j}$ 's).

Our next goal is to describe a set of moves which connect any two Klinks describing 4-thickenings with 2-equivalent spines or 2-equivalent decorated spines. We recall that according to Corollary 5.7.2 in [7], the second Whitney class $w_{2}(M)$ of a 4 -thickening $(M, P)$ is represented by a cocycle $\bar{c} \in H^{2}\left(M, M_{1}, \mathbb{Z} / 2\right) \simeq H^{2}\left(P, P_{1}, \mathbb{Z} / 2\right)$ whose value on each 2 -handle is its framing coefficient modulo 2 . In other words, if $M=M_{L}$, then the framing coefficient of the $i$ th undotted component of $L$ is equal to $\bar{c}_{i}$ modulo 2 .

2.5. Definition. Let $L$ be a K-link described with the corresponding regular plane projection. We say that $L^{\prime}$ is sw-equivalent to $L$ if it can be obtained from $L$ by a finite sequence of the following moves:

(i) 2-deformation;

(ii) changing the signs of a finite number of crossings of undotted segments.

$L^{\prime}$ is said to be s-equivalent to $L$ if it can be obtained from $L$ by a finite sequence of moves of the type (i), (ii) and

(iii) adding a positive or negative twist on an undotted component. 
2.6. Proposition. Two K-links $L$ and $L^{\prime}$ are s-equivalent if and only if $\left[P_{L}\right]=\left[P_{L^{\prime}}\right]$, and they are sw-equivalent if and only if $\left[P_{L}, \iota_{M, P}^{*}\left(w_{2}\left(M_{L}\right)\right)\right]=$ $\left[P_{L^{\prime}}, \iota_{M^{\prime}, P^{\prime}}^{*}\left(w_{2}\left(M_{L^{\prime}}\right)\right)\right]$.

Proof. Observe that moves (ii) and (iii) do not change $\widehat{P}_{L}$. Moreover, (ii) either leaves the framings of the corresponding undotted components unchanged, or changes them by \pm 2 . Hence, if $L$ and $L^{\prime}$ are $s$-equivalent (resp. sw-equivalent), then $M_{L}$ and $M_{L^{\prime}}$ have 2-equivalent spines (resp. decorated spines).

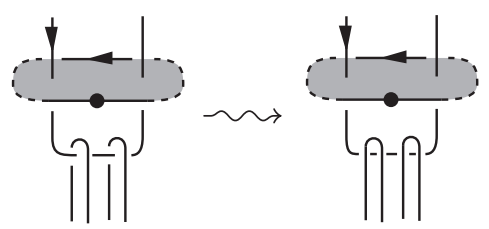

Fig. 4. Unknotting a segment

To see the opposite direction, according to 2.4, it is enough to show that if there exist BOK-links $\widehat{L}$ and $\widehat{L}^{\prime}$ and a 2-deformation $\xi: \widehat{P}_{L} \rightsquigarrow \widehat{P}_{L^{\prime}}$ which is just a sequence of cancellations of terms $x_{i} x_{i}^{-1}$ in the relations, then $L$ can be transformed into $L^{\prime}$ through regular isotopy and moves (ii) and (iii), where (iii) is needed only if the corresponding components of $L$ and $L^{\prime}$ have different framings modulo 2 . But a term $x_{i} x_{i}^{-1}$ in $\widehat{P}_{L}$ corresponds in $\widehat{L}$ to a segment of an undotted component which is limited by a negative and positive intersection with the Seifert surface of the $i$ th dotted component, $S_{i}$, and does not intersect any other Seifert surface. Then by changing crossings, we can unknot this segment from the other unknotted components and cancel its intersections with $S_{i}$ through regular isotopy (Figure 4). Observe that this operation leaves the framing of the undotted components the same modulo 2. After doing this to all such segments we obtain a K-link $\widehat{L}^{\prime \prime}$ with $\widehat{P}_{L^{\prime \prime}}=\widehat{P}_{L^{\prime}}$. Now the statement would follow from the one with $\xi=$ id. For $\xi=$ id it has been proved in [4, Lemma 2.16] by showing that if the corresponding undotted components of $L$ and $L^{\prime}$ have the same framings modulo 2, then through regular isotopy and move (ii) both diagrams can be deformed into the same standard form as a closure, through the dotted components, of a chosen braid. If the framings differ modulo 2 , we need move (iii) to adjust them.

2.7. Two K-links describe 4-thickenings with diffeomorphic boundaries if and only if they can be transformed into each other through a finite sequence of 2-deformation moves as in 2.3 and two extra moves (see [12]):

(d) removing (or adding) a dot on a zero framed unknot. This corresponds to replacing a 1-handle with its canceling 2-handle and vice versa; 
(e) deleting or adding an unknot $U^{ \pm 1}$ of framing \pm 1 , contained in a neighborhood ball in the rest of the link, which corresponds to taking a connected union with $\pm \mathbb{C} P^{2}$.

Let $M$ be a 4 -thickening represented by a K-link $L$ and let $\sigma_{+}, \sigma_{-}$and $\sigma_{0}$ be the numbers of positive, negative and zero eigenvalues of the linking matrix of $L$. Then index $(M)=\sigma_{+}-\sigma_{-}$and $\operatorname{rank} H_{1}(\partial M, \mathbb{Z})=\sigma_{0}$.

2.8. Definition. A $\mathcal{K}$-valued invariant $\mathcal{Z}_{\mathcal{K}}^{4}$ of 4 -thickenings is called modular if it is multiplicative with respect to connected sum and there exists $X \neq 0 \in \mathcal{K}$, not a zero divisor, such that if $L$ is a K-link obtained from $L^{\prime}$ by removing a dot from a component then $\mathcal{Z}_{\mathcal{K}}^{4}\left(M_{L}\right)=X \mathcal{Z}_{\mathcal{K}}^{4}\left(M_{L^{\prime}}\right)$.

2.9. Proposition. Let $\mathcal{Z}_{\mathcal{K}}^{4}$ be a $\mathcal{K}$-valued modular invariant of 4 -thickenings and let $C_{+}=\mathcal{Z}_{\mathcal{K}}^{4}\left(\mathbb{C} P^{2}\right)$ and $C_{-}=\mathcal{Z}_{\mathcal{K}}^{4}\left(-\mathbb{C} P^{2}\right)$. Then

(a) $C_{+} C_{-}=X$. In particular

$$
C_{+}^{n-\sigma_{+}} C_{-}^{n-\sigma_{-}}=\left(\frac{C_{+}}{C_{-}}\right)^{-\operatorname{index}(M) / 2} X^{\left(1+\sigma_{0}-\chi(M)\right) / 2}
$$

is an invariant of 4 -thickenings.

(b) Let $\mathcal{K}^{\prime}=\mathcal{K}\left[1 / C_{-}, 1 / C_{+}\right]$. Then for any 4-thickening $M$ represented by a K-link $L$ with $n$ dotted components, $C_{+}^{n-\sigma_{+}} C_{-}^{n-\sigma_{-}} \mathcal{Z}_{\mathcal{K}}^{4}(M) \in \mathcal{K}^{\prime}$ depends only on the boundary $\partial M$ of $M$ and is denoted by $\mathcal{Z}_{\mathcal{K}}^{\partial}(\partial M)$.

Proof. (a) follows from the fact that the connected sum $\mathbb{C} P^{2} \#-\mathbb{C} P^{2}$ is related to $B^{4}$ by move $2.3(\mathrm{c})$ and $2.5(\mathrm{~d})$ as shown in Figure 5. Moreover, since it is a modular invariant, under moves $2.5(\mathrm{~d})$ and $(\mathrm{e}), \mathcal{Z}_{\mathcal{K}}^{4}(M)$ changes exactly as $C_{+}^{\sigma_{+}-n} C_{-}^{\sigma_{-}-n}$ and therefore the quotient of those two invariants depends only on the boundary of $M$.

Observe that the proposition implies that a modular invariant is actually an invariant of smooth 4-manifolds which have a decomposition into 0-, 1and 2-handles.

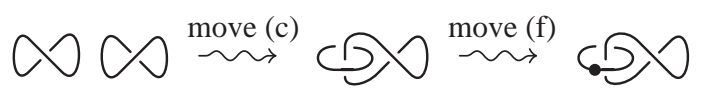

Fig. 5. $\mathbb{C} P^{2} \#-\mathbb{C} P^{2}$ is related to $B^{4}$ by move (f)

2.10. Definition. An $\mathcal{R}$-valued invariant of 2 -complexes (resp. decorated 2-complexes) $\mathcal{Z}_{\mathcal{R}}^{2}$ is called a reduction of a $\mathcal{K}$-valued invariant $\mathcal{Z}_{\mathcal{K}}^{4}$ of 4 -thickenings if there exists a ring homomorphisms $\varphi: \mathcal{K} \rightarrow \mathcal{R}$ such that for any 4 -thickening $(M, P), \mathcal{Z}_{\mathcal{K}}^{4}(M) \otimes_{\varphi} \mathcal{R}=\mathcal{Z}_{\mathcal{R}}^{2}(P)$ (resp. $\mathcal{Z}_{\mathcal{K}}^{4}(M) \otimes_{\varphi} \mathcal{R}=$ $\mathcal{Z}_{\mathcal{R}}^{2}\left(P, \iota_{M, P}^{*}\left(w_{2}(M)\right)\right)$. 
2.11. TheOREM. Let $\mathcal{Z}_{\mathcal{R}}^{2}$ be an invariant of 2-complexes which is a reduction of a modular invariant $\mathcal{Z}_{\mathcal{K}}^{4}$. Then if $P$ is a 2-dimensional $C W$ complex with $\chi(P) \geq 1, \mathcal{Z}_{\mathcal{R}}^{2}(P)$ depends only on the homology of $P$. In particular, if $H_{1}(P)=\left(\bigoplus_{i} \mathbb{Z} / t_{i}\right) \oplus \mathbb{Z}^{b_{1}}$ and $H_{2}(P)=\mathbb{Z}^{b_{2}}$ then

$$
\mathcal{Z}_{\mathcal{R}}^{2}(P)=\varphi\left(X^{-b_{1}} Y_{0}^{b_{2}+b_{1}} \prod_{i} Y_{t_{i}} Y_{-t_{i}}\right)
$$

where $Y_{n}=\mathcal{Z}_{\mathcal{K}^{\prime}}^{\partial}(L(n, 1))$ is the invariant of the corresponding lens space.

Proof. Let $\mathrm{P}\left[b_{1}, b_{2},\left\{t_{i}\right\}_{i}\right]$ denote the one-point union $\left(\bigvee_{i} \Omega_{t_{i}}\right) \vee\left(\bigvee^{b_{1}} S^{1}\right) \vee$ $\left(\bigvee^{b_{2}} S^{2}\right)$, where $\Omega_{t}$ is the 2-complex with $\widehat{\Omega}_{t}=\left\langle x \mid x^{t}\right\rangle$. We will first see that the formula holds for $\mathrm{P}\left[b_{1}, b_{2},\left\{t_{i}\right\}_{i}\right]$. Observe that since $\mathcal{Z}_{\mathcal{K}}^{4}$ is multiplicative with respect to connected sum, its reduction $\mathcal{Z}_{\mathcal{R}}^{2}$ is multiplicative with respect to one-point union. Moreover, Proposition 2.9(b) implies that $\mathcal{Z}_{\mathcal{R}}^{2}\left(S^{2}\right)=\varphi\left(\mathcal{Z}_{\mathcal{K}}^{4}\left(S^{2} \times D^{2}\right)\right)=\varphi\left(Y_{0}\right)$ and $\mathcal{Z}_{\mathcal{R}}^{2}\left(S^{1}\right)=\varphi\left(\mathcal{Z}_{\mathcal{K}}^{4}\left(S^{1} \times D^{3}\right)\right)=$ $\varphi\left(Y_{0} / X\right)$. Let now $\left(M_{t}, \Omega_{t}\right), t>0$, be the 4 -thickening described by the K-link of Figure 6(a). Figure 6(b) has been obtained from (a) by deleting the dot from the unknot and sliding it over the other component, and therefore describes $\partial M_{t}$ as $Y_{t} \# Y_{-t}$. Since $\sigma_{ \pm}\left(M_{t}\right)=1$, from 2.9(b) it follows that

$$
\mathcal{Z}_{\mathcal{R}}^{2}\left(\Omega_{t}\right)=\varphi\left(\mathcal{Z}_{\mathcal{K}}^{4}\left(M_{t}\right)\right)=\varphi\left(Y_{t} Y_{-t}\right)
$$

Hence the formula holds for the complex $\mathrm{P}\left[b_{1}, b_{2},\left\{t_{i}\right\}_{i}\right]$.

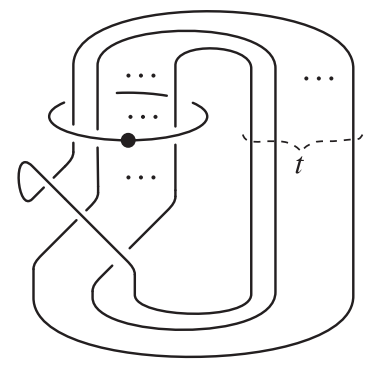

(a)

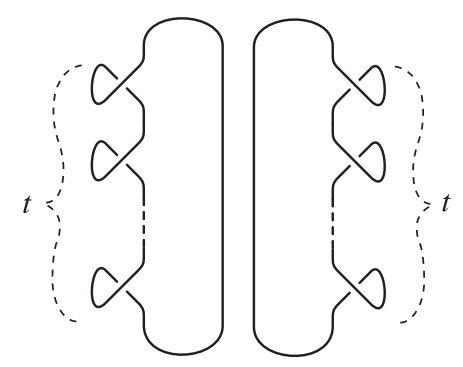

(b)

Fig. 6. The K-link corresponding to the cyclic group

The general statement follows from Corollary 1.5 in [17] which states that two complexes $P$ and $P^{\prime}$ occur (up to 2-deformation) as dual spines of some Heegard decomposition of $S^{4}$ if and only if they are connected and satisfy the Alexander-Lefschetz duality: $H_{1}(P) \simeq H^{2}\left(P^{\prime}\right)$ and $H_{2}(P) \simeq H^{1}\left(P^{\prime}\right)$ (this is a generalization of a result of Huck [9] concerning acyclic complexes). In particular, if $H_{1}(P)=\left(\bigoplus_{i} \mathbb{Z} / t_{i}\right) \oplus \mathbb{Z}^{b_{1}}$ and $H_{2}(P)=\mathbb{Z}^{b_{2}}$, there exists a decomposition $S^{4}=M \cup_{\partial} \overline{M^{\prime}}$, as a union along the boundary, of a 4- 
thickening $M$ of a complex 2-equivalent to $P$ and a 4-thickening $M^{\prime}$ of a complex 2-equivalent to $P^{\prime}=\mathrm{P}\left[b_{2}, b_{1},\left\{t_{i}\right\}_{i}\right]$. Here $\overline{M^{\prime}}$ indicates $M^{\prime}$ with the reversed orientation. Since index $\left(S^{4}\right)=0$, we have $\operatorname{index}(M)=\operatorname{index}\left(M^{\prime}\right)$. Moreover Proposition 2.9(b) implies that

$$
\begin{aligned}
\mathcal{Z}_{\mathcal{K}^{\prime}}^{\partial}(\partial M) & =\left(\frac{C_{+}}{C_{-}}\right)^{-\operatorname{index}(M) / 2} X^{\left(1+\sigma_{0}-\chi(M)\right) / 2} \mathcal{Z}_{\mathcal{K}}^{4}(M) \\
& =\left(\frac{C_{+}}{C_{-}}\right)^{-\operatorname{index}\left(M^{\prime}\right) / 2} X^{\left(1+\sigma_{0}-\chi\left(M^{\prime}\right)\right) / 2} \mathcal{Z}_{\mathcal{K}}^{4}\left(M^{\prime}\right) .
\end{aligned}
$$

Hence $\mathcal{Z}_{\mathcal{K}}^{4}(M)=X^{\chi(P)-1} \mathcal{Z}_{\mathcal{K}}^{4}\left(M^{\prime}\right)$ and if $\chi(P) \geq 1$ then

$$
\mathcal{Z}_{\mathcal{R}}^{2}(P)=\varphi\left(\mathcal{Z}_{\mathcal{K}}^{4}(M)\right)=\varphi\left(X^{\chi(P)-1}\right) \mathcal{Z}_{\mathcal{R}}^{2}\left(P^{\prime}\right)=\varphi\left(X^{-b_{1}} Y_{0}^{b_{2}+b_{1}} \prod_{i} Y_{t_{i}} Y_{-t_{i}}\right)
$$

\section{Invariants of 4-thickenings constructed from finite semisimple tortile categories}

3.1. A finite semisimple category $\mathcal{C}$ (see for example [15]) is an additive category over a ring $\mathcal{K}$ such that there is a finite set $S$ of simple objects and any other object is isomorphic to a finite sum of copies of those, and for any $a, b \in S, \operatorname{hom}(a, b)$ is 0 if $a \neq b$ and is naturally isomorphic to $\mathcal{K}$ if $a=b$. We will use small letters $a, b, \ldots$ to denote elements in $S$. Observe that for any object $A$ in $\mathcal{C}$ and any $a \in S$, the $\mathcal{K}$-modules $\operatorname{hom}(a, A)$ are finitedimensional and we will denote by $\left\{\varepsilon_{i}(a, A)\right\}_{i}$ a basis for these modules, omitting $(a, A)$ when they are clear from the context. Moreover, the map $\operatorname{hom}(A, a) \rightarrow \operatorname{hom}(a, A)^{*}$ given by composition and the natural isomorphism $\operatorname{hom}(a, a) \simeq \mathcal{K}$ is an isomorphism, and we will denote by $\left\{\bar{\varepsilon}_{i}(A, a)\right\}_{i}$ (or simply $\left.\left\{\bar{\varepsilon}_{i}\right\}_{i}\right)$ the basis of $\operatorname{hom}(A, a)$ dual to $\left\{\varepsilon_{i}(a, A)\right\}_{i}$.

3.2. A semisimple tortile category $\mathcal{C}$ (see [20]) is a finite semisimple category over $\mathcal{K}$ which first of all is equipped with a product $\mathcal{C} \times \mathcal{C} \rightarrow \mathcal{C}$ with identity $\mathbf{1} \in S$, and also any object $A$ is equipped with a right dual $\bar{A}$. The product will be denoted by $\diamond$ or simply by juxtaposition: $A \diamond B=A B$. For any objects $A, B, C$ we are given the following set of morphisms:

$$
\begin{array}{cl}
\varrho_{A}: A \rightarrow \mathbf{1} A, & \varrho_{A}^{-1}: \mathbf{1} A \rightarrow A, \\
\alpha_{A, B, C}:(A B) C \rightarrow A(B C), & \alpha_{A, B, C}^{-1}: A(B C) \rightarrow(A B) C \text { (associativity), } \\
\gamma_{A, B}: A B \rightarrow B A, & \gamma_{A, B}^{-1}: B A \rightarrow A B \text { (commutativity), } \\
\Lambda_{A}: \mathbf{1} \rightarrow \bar{A} A \text { (coform), } & \lambda_{A}: A \bar{A} \rightarrow \mathbf{1} \text { (form), }
\end{array}
$$

which satisfy a number of identities described for example in [20]. Based on the coherence result of MacLane for monoidal categories (p. 161 in [15]), in what follows we will often omit the associativity morphisms since they can be filled in a unique way. Below we define and list some basic properties 
of the twist, rank and projection operators in a semisimple tortile category. We refer the reader to $[20,22,19]$ for proofs and details.

3.3. The twist $\theta_{A}=\left(\lambda_{A} \diamond \mathrm{id}_{A}\right) \circ\left(\mathrm{id}_{\bar{A}} \diamond \gamma_{A, A}\right) \circ\left(\Lambda_{A} \diamond \mathrm{id}_{A}\right)$ is a natural isomorphism $A \simeq A$ with $\theta_{A}^{-1}$ given by the same expression with $\gamma_{A, A}$ replaced by $\gamma_{A, A}^{-1}$. In particular, $\theta_{B}^{-1} \psi \theta_{A}=\psi$ for any $\psi \in \operatorname{hom}(A, B)$. Moreover, $\theta_{a b}=\theta_{a}^{-1} \theta_{b}^{-1} \gamma_{b, a} \gamma_{a, b}$ for any $a, b \in S$.

The choice of a twist is equivalent to the choice of a natural monoidal isomorphism $\overline{\bar{A}} \rightarrow A$ (Proposition 2.4 in [22]). Combining with these isomorphisms if necessary, in what follows we will assume that "_" is an involution on the set of objects and on $S$, and that $\overline{A \diamond B}=\bar{B} \diamond \bar{A}$ with $\lambda_{A B}=\lambda_{A} \circ\left(\operatorname{id}_{A} \diamond \lambda_{B} \diamond \mathrm{id}_{\bar{A}}\right)$ and $\Lambda_{A B}=\left(\operatorname{id}_{\bar{A}} \diamond \Lambda_{B} \diamond \mathrm{id}_{A}\right) \circ \Lambda_{A}$.

3.4. For any $a \in S$ and $A \in \operatorname{Obj}(\mathcal{C})$ define

$$
\pi(a, A)=\sum_{i} \varepsilon_{i}(a, A) \circ \bar{\varepsilon}_{i}(A, a) \in \operatorname{hom}(A, A) .
$$

Then $\pi(a, A)$ is a projection operator independent of the choice of the basis $\left\{\varepsilon_{i}\right\}_{i}$, and $\sum_{a \in S} \pi(a, A)=\mathrm{id}_{A}$. These properties together with the semisimplicity of the category imply that for any $\psi \in \operatorname{hom}(A, B)$ and $a \in S$,

$\psi \circ \pi(a, A)=\sum_{b \in S} \pi(b, B) \circ \psi \circ \pi(a, A)=\pi(a, B) \circ \psi \circ \pi(a, A)=\pi(a, B) \circ \psi$.

3.5. The rank of a morphism $f \in \operatorname{hom}(A, A)$ is given by $\operatorname{rank}(f)=$ $\lambda_{\bar{A}}(\mathbf{1} \diamond f) \Lambda_{A} \in \operatorname{hom}(\mathbf{1}, \mathbf{1}) \simeq \mathcal{K}$, and the rank of an object $A \in \operatorname{Obj}(\mathcal{C})$ is $r_{A}=\operatorname{rank}\left(\operatorname{id}_{A}\right)$. Moreover, given any $f \in \operatorname{hom}(A, B), g \in \operatorname{hom}(B, A)$ and $h \in \operatorname{hom}(C, D)$,

$$
\operatorname{rank}(f \circ g)=\operatorname{rank}(g \circ f) \quad \text { and } \operatorname{rank}(f \diamond h)=\operatorname{rank}(f) \operatorname{rank}(h) .
$$

In particular $r_{A B}=r_{A} r_{B}$. In what follows we will assume $\left({ }^{5}\right)$ that $r_{a} \neq 0$ for any $a \in S$. In particular, using the natural isomorphisms $\operatorname{hom}(a, a) \simeq \mathcal{K}$, we see that $f=\left(\operatorname{rank}(f) / r_{a}\right) \operatorname{id}_{a}$ for any $f \in \operatorname{hom}(a, a)$. Moreover,

$$
\pi(\mathbf{1}, a b)= \begin{cases}0 & \text { if } b \neq \bar{a}, \\ \left(1 / r_{a}\right) \Lambda_{\bar{a}} \circ \lambda_{a} & \text { otherwise. }\end{cases}
$$

3.6. Proposition. Let

$$
\begin{aligned}
& \widehat{\zeta}_{i}(\bar{b} a, c)=\left(\lambda_{\bar{b}} \diamond \mathrm{id}_{c}\right) \circ\left(\operatorname{id}_{\bar{b}} \diamond \varepsilon_{i}(a, b c)\right), \\
& \zeta_{i}(c, \bar{b} a)=\left(\operatorname{id}_{\bar{b}} \diamond \bar{\varepsilon}_{i}(b c, a)\right) \circ\left(\Lambda_{b} \diamond \operatorname{id}_{c}\right) .
\end{aligned}
$$

Then $\left\{\zeta_{i}\right\}_{i}$ forms a basis for $\operatorname{hom}(c, \bar{b} a)$ with dual basis $\bar{\zeta}_{i}=\left(r_{c} / r_{a}\right) \widehat{\zeta}_{i}$.

$\left({ }^{5}\right)$ If necessary one can eliminate objects of zero rank taking the quotient category by all morphisms which factor through such objects $[19,6]$. 
Proof. From the properties of the rank in 3.5 it follows that $\widehat{\zeta}_{i} \circ \zeta_{j}=\frac{1}{r_{c}} \operatorname{rank}\left(\widehat{\zeta}_{i} \circ \zeta_{j}\right)=\frac{1}{r_{c}} \operatorname{rank}\left(\varepsilon_{i} \circ \bar{\varepsilon}_{j}\right)=\frac{1}{r_{c}} \operatorname{rank}\left(\bar{\varepsilon}_{j} \circ \varepsilon_{i}\right)=\frac{r_{a}}{r_{c}} \delta_{i, j}$.

3.7. Proposition. Let $\delta: \operatorname{hom}(A, B) \rightarrow \operatorname{hom}(\bar{B}, \bar{A})$ be given by

$$
\delta(\varphi)=\left(\operatorname{id}_{\bar{A}} \diamond \lambda_{B}\right) \circ\left(\operatorname{id}_{\bar{A}} \diamond \varphi \diamond \operatorname{id}_{\bar{B}}\right) \circ\left(\Lambda_{A} \diamond \mathrm{id}_{\bar{B}}\right) .
$$

Then

(a) $\lambda_{B} \circ\left(\varphi \diamond \operatorname{id}_{\bar{B}}\right)=\lambda_{A} \circ\left(\operatorname{id}_{A} \diamond \delta(\varphi)\right)$ and $\left(\operatorname{id}_{\bar{A}} \diamond \varphi\right) \circ \Lambda_{A}=\left(\delta(\varphi) \diamond \operatorname{id}_{B}\right) \circ \Lambda_{B}$.

(b) $\delta^{2}=\mathrm{id}$. In particular, $\delta$ is an isomorphism.

(c) $\overline{\delta\left(\bar{\varepsilon}_{i}(b c, a)\right)}=\delta\left(\varepsilon_{i}(a, b c)\right)$.

Proof. (a) and (c) are direct consequences of the axioms for the form and coform. The naturality of the commutativity morphism implies that $\delta^{2}(\varphi)=\theta_{B}^{-1} \varphi \theta_{A}$. Then (b) follows from 3.3 .

3.8. We define the category of $\mathrm{K}$-tangles labeled in $\mathcal{C}, \mathcal{T}(\mathcal{C})$, to be the category with objects sets of points in $\mathbb{R}^{2}$, labeled by elements in $S$, and morphisms compositions of products of elementary tangles presented in Figure 7. The relations in the category are given by the consistently labeled Reidemeister moves and moves presented in Figure 3. We observe that if $T$ is a $\mathrm{K}$-tangle with fixed orientation on each undotted component, and if $\mu$ is a map from the set of undotted components into $S$, then there exists a unique labeled tangle $T(\mu)$ which has $T$ as underlying tangle such that the upgoing segments of $x$ are labeled by $\operatorname{id}_{\mu(x)}$. Moreover, $F(T(\mu))$ is independent of the orientation of the closed components in $T$.

(a)

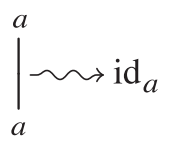

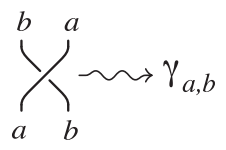
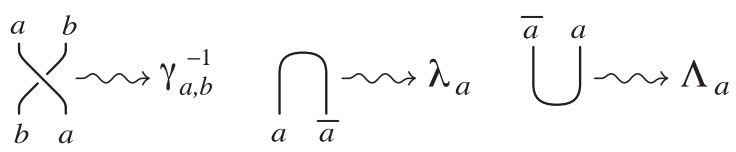

(b)

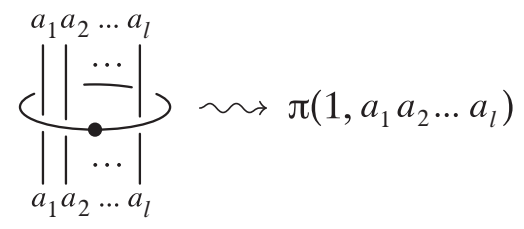

Fig. 7. Defining the functor $F$

Following the generalization of Kerler $[10,11]$ of the results of Reshetikhin-Turaev and Shum [19, 20] we define a monoidal braided functor $F$ from $\mathcal{T}(\mathcal{C})$ to $\mathcal{C}$ where the images of the elementary tangles are presented in Figure 7 . To see that $F$ is a functor the only additional relations to check (in view of the coherence result in [20]) are the ones presented in Figure 3: (d) and (e) there follow from the naturality of the braiding, (a) and (b) follow 
from the naturality of $\pi(a, A)$ in 3.4, and (c) follows from Proposition 3.7(a) and the fact that $\delta(\pi(1, A))=\pi(1, \bar{A})$.

Let $L$ be a K-link with $n$ dotted and $m$ undotted components and let $\mu$ be a labeling of $L$ such that $a_{i} \in S$ is the image of the $i$ th undotted component. Define

$$
\{L\}=\sum_{\mu} w(\mu) F(L(\mu)),
$$

where $w(\mu)=r_{a_{1}} \cdots r_{a_{m}}$ and the sum is over all possible labelings of $L$.

3.9. TheOREM. Let $M$ be a 4-thickening represented by a K-link L. Then $\{L\}$ defines an invariant of $M$ under 2-deformations, which will be denoted by $\mathcal{Z}_{\mathcal{K}}^{4}(M)$.

Observe that this implies that when $L$ does not have dotted components, $\{L\}$ is a link invariant under the connected sum of two undotted components (the first Kirby move) without putting any modularity condition on the category. This is exactly Proposition 1.1 in [2]. As we will see later, the modularity condition is needed for the invariance under the second Kirby move, i.e. adding or deleting an unknot of framing \pm 1 . The proof below follows the idea in [2] and is presented for the sake of completeness.

Proof. According to 2.3 it is enough to show the invariance under

(a) adding or deleting a canceling pair of a dotted and undotted component;

(b) connected sum of two undotted components.

(a) follows from the fact that if a single undotted component $y$ intersects once the Seifert surface of a dotted component $x$ then the only label of $y$, giving a non-trivial contribution to $\{L\}$, is $\mathbf{1}$.

To prove (b) suppose that we want to slide the undotted component $x$ over the undotted component $y$. Then $L$ can be presented as the closure of a 2-2 tangle $T$ on these two components. Let $\mu_{a, b}$ be a labeling of $T$ where $x$ is labeled by $a$ and $y$ is labeled by $b$ and let $w^{\prime}\left(\mu_{a, b}\right)=w\left(\mu_{a, b}\right) / r_{a} r_{b}$. Then

$$
\{L\}=\sum_{a, b} r_{a} r_{b} \operatorname{rank}\left(\psi_{a, b}\right), \quad \text { where } \quad \psi_{a, b}=\sum_{\mu_{a, b}} w^{\prime}\left(\mu_{a, b}\right) F\left(T\left(\mu_{a, b}\right)\right),
$$

and the sum is over all possible values of $\mu_{a, b}$. In terms of labeled tangle diagrams the following steps in the proof are illustrated in Figure 8 and are explained below.

Let $T^{\#}$ denote the 3-3 tangle which is obtained from $T$ by adding a parallel component $y^{\prime}$ of $y$. After the sliding of $x$ over $y$ the resulting link $L^{\#}$ can be thought of as obtained from the closure of $T^{\#}$ connecting $x$ and $y^{\prime}$ by a small band. Let now $\mu_{a, b}^{\#}$ denote the labeling of $L^{\#}$ induced by $\mu_{a, b}$ 


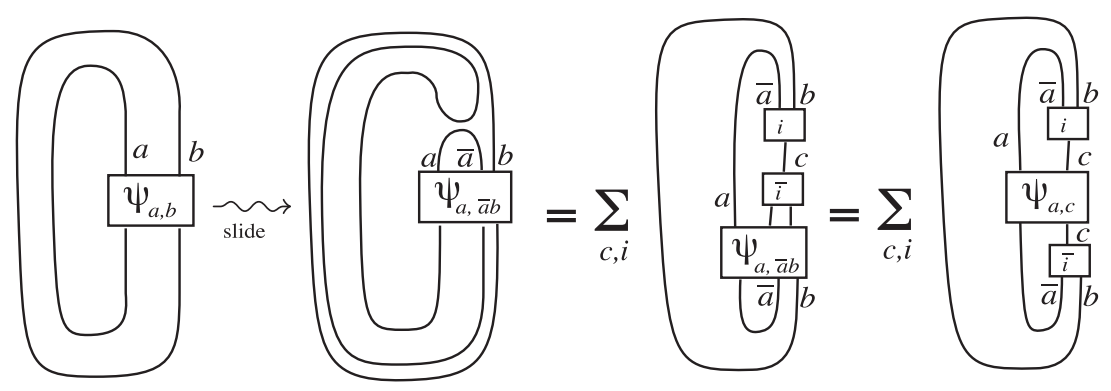

Fig. 8. 2-handle slide

and let $\psi_{a, \bar{a} b}=\sum_{\mu_{a, b}^{\#}} w^{\prime}\left(\mu_{a, b}\right) F\left(T^{\#}\left(\mu_{a, b}^{\#}\right)\right)$. Then

$$
\begin{aligned}
\left\{L^{\#}\right\} & =\sum_{a, b} r_{a} r_{b} \operatorname{rank}\left(\left(\Lambda_{\bar{a}} \diamond \mathrm{id}_{b}\right) \circ\left(\lambda_{a} \diamond \mathrm{id}_{b}\right) \circ \psi_{a, \bar{a} b}\right) \\
& =\sum_{a, b} r_{a} r_{b} \operatorname{rank}\left(\left(\lambda_{a} \diamond \mathrm{id}_{b}\right) \circ \psi_{a, \bar{a} b} \circ\left(\Lambda_{\bar{a}} \diamond \mathrm{id}_{b}\right)\right) \\
& =\sum_{a, b, c, i} r_{a} r_{b} \operatorname{rank}\left(\left(\lambda_{a} \diamond \mathrm{id}_{b}\right)\right. \\
& \left.=\sum_{a, b, c, i} r_{a} r_{b} \operatorname{rank}\left(\widehat{\zeta}_{i}(a c, b) \circ \varepsilon_{i}(c, \bar{a} b)\right) \circ \psi_{a, c} \circ\left(\operatorname{id}_{a} \diamond \bar{\varepsilon}_{i}(\bar{a} b, c)\right) \circ\left(\Lambda_{\bar{a}} \diamond \mathrm{id}_{b}\right)\right)
\end{aligned}
$$

where $\widehat{\zeta}_{i}(a c, b)$ and $\zeta_{i}(b, a c)$ are defined in 3.6. Then Proposition 3.6 implies that

$$
\begin{aligned}
\left\{L^{\#}\right\} & =\sum_{a, c, b, i} r_{c} r_{a} \operatorname{rank}\left(\bar{\zeta}_{i} \circ \psi_{a, c} \circ \zeta_{i}\right)=\sum_{a, c} r_{c} r_{a} \operatorname{rank}\left(\sum_{b, i}\left(\zeta_{i} \circ \bar{\zeta}_{i}\right) \circ \psi_{a, c}\right) \\
& =\sum_{a, c} r_{c} r_{a} \operatorname{rank}\left(\sum_{b}\left(\pi(b, a c) \circ \psi_{a, c}\right)\right)=\sum_{a, c} r_{c} r_{a} \operatorname{rank}\left(\psi_{a, c}\right)=\{L\},
\end{aligned}
$$

where we have used 3.4 and 3.5.

The case of a modular category. Let $H(a, b)$ denote the Hopf link where one component has been labeled with $a$ and the other with $b$ and let $S_{a, b}=$ $F(H(a, b))$. Then $\mathcal{C}$ is called modular if there exists $X \neq 0 \in \mathcal{K}$, not a zero divisor, such that $\sum_{b} r_{b} S_{a, b}=X \delta_{a, 1}$. Lemma 17 in Section 4.2 of [10] shows that this definition over a field is equivalent to the usual modularity condition, i.e. the invertibility of the $S$-matrix.

Let $\dot{\Phi}_{l}\left(a_{1}, \ldots, a_{l}\right)$ be the K-tangle consisting of $l$ vertical segments, labeled by $a_{i} \in S$, and one unknotted dotted closed component which goes around them as presented in Figure $7(\mathrm{~b})$. Let also $\Phi_{l}\left(b, a_{1}, \ldots, a_{l}\right)$ be the same tangle where the dotted unknot has been replaced with an undotted 
one and it has been labeled by $b \in S$. Then the semisimplicity and modularity of the category imply that

$$
\begin{aligned}
\sum_{b} r_{b} F\left(\Phi_{l}\left(b, a_{1}, \ldots, a_{l}\right)\right) & =\sum_{b} r_{b} S_{a, b} \pi\left(a, a_{1} \cdots a_{l}\right) \\
& =X \pi\left(\mathbf{1}, a_{1} \cdots a_{l}\right)=X F\left(\dot{\Phi}_{l}\left(a_{1}, \ldots, a_{l}\right)\right) .
\end{aligned}
$$

Hence we have

3.10. Proposition. If $\mathcal{C}$ is a modular tortile category then $\mathcal{Z}_{\mathcal{K}}^{4}(M)$ is a modular invariant with $X=\mathcal{Z}_{\mathcal{K}}^{4}\left(S^{2} \times D^{2}\right)=\sum_{a \in S} r_{a}^{2}$.

The case of a symmetric category. A finite semisimple tortile category is called symmetric if $\gamma_{b, a} \gamma_{a, b}=\mathrm{id}_{a b}$.

3.11. Proposition. A finite semisimple tortile category is symmetric if and only if for any $a \in S, \theta_{a}=\nu_{a} \mathrm{id}_{a}$ where $\nu_{a}= \pm 1$, and for any triple $(a, b, c)$ such that $\operatorname{hom}(c, a b) \neq 0, \nu_{a} \nu_{b} \nu_{c}=1$.

Proof. The expressions for the twist and its inverse in 3.3 imply that in a symmetric category $\theta_{a}^{-1}=\theta_{a}$, i.e. $\theta_{a}=\nu_{a} \mathrm{id}_{a}$ where $\nu_{a}= \pm 1$. On the other hand, if $\mathcal{C}$ is a tortile category and $\theta_{a}=\nu_{a} \mathrm{id}_{a}, \theta_{a b}=\theta_{a}^{-1} \theta_{b}^{-1} \gamma_{b, a} \gamma_{a, b}$ implies that $\nu_{a} \nu_{b} \nu_{c} \mathrm{id} c_{c}=\bar{\varepsilon}_{i}(a b, c) \gamma_{b, a} \gamma_{a, b} \varepsilon_{i}(c, a b)$. Hence such a category is symmetric if and only if $\nu_{a} \nu_{b} \nu_{c}=1$ for any triple $(a, b, c)$ such that $\operatorname{hom}(c, a b) \neq 0$.

In $[18,3]$ it has been shown that from every finite semisimple symmetric category over a ring $\mathcal{K}$ such that $\nu_{a}=1$ for any $a \in S$, one can construct a $\mathcal{K}$ valued invariant of 2-dimensional $\mathrm{CW}$ complexes, called $Q_{\mathcal{K}}$. The following lemma is a slight generalization of this result for decorated 2-complexes.

3.12. Proposition. If $\mathcal{C}$ is a finite semisimple symmetric category over a ring $\mathcal{K}$, then for any 4 -thickenings $(M, P)$ and $\left(M^{\prime}, P^{\prime}\right)$ such that

$$
\left[P, \iota_{M, P}^{*}\left(w_{2}(M)\right)\right]=\left[P^{\prime}, \iota_{M^{\prime}, P^{\prime}}^{*}\left(w_{2}\left(M^{\prime}\right)\right)\right]
$$

we have $\mathcal{Z}_{\mathcal{K}}^{4}(M)=\mathcal{Z}_{\mathcal{K}}^{4}\left(M^{\prime}\right)$, i.e. $\mathcal{Z}_{\mathcal{K}}^{4}(M)$ defines an invariant of decorated 2 -complexes which will be denoted by $\mathcal{Z}_{\mathcal{K}}^{2}(P, w)$. If in addition $\nu_{a}=1$ for any $a \in S$, then $\mathcal{Z}^{2}(P, w)$ depends only on $P$ and will be denoted by $\mathcal{Z}_{\mathcal{K}}^{2}(P)$.

Proof. Let $M_{L}$ be described by a K-link $L$. Then $\gamma_{a, b}=\gamma_{b, a}^{-1}$ implies that the value of $\mathcal{Z}_{\mathcal{K}}^{4}\left(M_{L}\right)$ does not change if we change the sign of a crossing between two undotted components. Moreover under the functor $F$, the image of a twist labeled by $a \in S$ is exactly $\theta_{a}$. Hence if $\nu_{a}=1$, adding or removing such twists does not change the value of the invariant either. Now the statement follows from Proposition 2.6.

Let $\mathcal{C}$ be a finite semisimple category and let $s=|S|$. The map $A \rightarrow$ $\bigoplus_{a \in S} \operatorname{hom}(a, A)$ induces equivalence of $\mathcal{C}$ into the category $\mathcal{K}(S)$ with ob- 
jects

$$
A\left(n_{1}, \ldots, n_{s}\right)=\bigoplus_{i=1}^{s} \mathcal{K}^{n_{i}}
$$

and morphisms $A\left(n_{1}, \ldots, n_{s}\right) \rightarrow A\left(m_{1}, \ldots, m_{s}\right)$ described by block matrices in $\bigoplus_{i=1}^{s} \mathcal{K}^{n_{i} \times m_{i}}$ acting on the left. If in addition $\mathcal{C}$ is a tortile category, its structure morphisms induce a tortile structure on $\mathcal{K}(S)$ which can be described by presenting the list of the block matrices corresponding to the morphisms $\varrho, \alpha, \gamma, \Lambda$ and $\lambda$.

3.13. Definition. Let $\mathcal{C}$ be a finite semisimple tortile category over a commutative ring $\mathcal{K}$ and $\varphi: \mathcal{K} \rightarrow \mathcal{R}$ be a ring homomorphism. Then $\varrho \otimes \mathcal{R}, \alpha \otimes \mathcal{R}, \gamma \otimes \mathcal{R}, \Lambda \otimes \mathcal{R}$ and $\lambda \otimes \mathcal{R}$ induce on the category $\mathcal{R}(S)=$ $\mathcal{K}(S) \otimes_{\varphi} \mathcal{R}$ the structure of a finite semisimple tortile category. If $\mathcal{R}(S)$ is symmetric it is called an $\mathcal{R}$-reduction of $\mathcal{C}$.

3.14. COROLlary. If a symmetric category is an $\mathcal{R}$-reduction of a finite semisimple tortile category over $\mathcal{K}$ then $\mathcal{Z}_{\mathcal{K}}^{4} \otimes_{\varphi} \mathcal{R}=\mathcal{Z}_{\mathcal{R}}^{2}$ is an $\mathcal{R}$-reduction of $\mathcal{Z}_{\mathcal{K}}^{4}$

4. Example: reduction in the $\mathrm{so}(3)$ case. Let $p=2 l+3, l \geq 1$, be an odd prime, $\mathcal{K}=\mathbb{Z}\left[v, v^{-1}\right]$, and $q_{p}=\frac{v^{p}-1}{v-1} \in \mathcal{K}$. Let also $\mathcal{K}_{p}$ be the quotient of $\mathcal{K}$ by the ideal generated by $q_{p}$, and let $\mathcal{K}_{p}^{\prime}$ be the fraction field of $\mathcal{K}_{p}$. In what follows we will use the common notation for the quantum integers

$$
[n]=\frac{v^{n}-v^{-n}}{v-v^{-1}} .
$$

4.1. Definition. A finite semisimple tortile category $\mathcal{C}$ over $\mathcal{K}_{p}$ will be called of so(3) type if it satisfies the following conditions:

(i) it has $l+1$ simple objects which will be identified with elements of $S=\{a \in \mathbb{Z} \mid 0 \leq a \leq l\}$

(ii) $\operatorname{hom}(a, b \diamond c) \simeq \mathcal{K}_{p}$ if all of the conditions below are satisfied:

$a+b+c \leq p-2, \quad a+b-c \geq 0, \quad a+c-b \geq 0, \quad b+c-a \geq 0$.

Otherwise $\operatorname{hom}(a, b \diamond c)=0$.

(iii) $r_{a}=[2 a+1]$ and $\theta_{a}=v^{-2 a(a+1)}$.

Whenever the so(3) Gelfand-Kazhdan category [6] is defined over the ring $\mathcal{K}_{p}$ it is an so(3) type category. A numerical procedure generating such categories and the smallest example corresponding to $p=5$ is presented in Section 5.

Let $U_{n}$ be the unknot of framing $n$ and let $\bar{n}$ denote the $\bmod p$ inverse of $n$. Then from the definition of $\{L\}$ we find that for such a category, 


$$
\begin{aligned}
\left\{U_{n}\right\}=\sum_{a=0}^{l}[2 a+1]^{2} & v^{-2 a n(a+1)} \\
& = \begin{cases}\left(\frac{-\overline{2} n}{p}\right) \frac{g_{1} v^{\left(n^{2}+2\right) / 2 n}}{\left(v-v^{-1}\right)}[\bar{n}] & \text { if } n \text { is prime to } p, \\
-\frac{p}{\left(v-v^{-1}\right)^{2}} & \text { otherwise. }\end{cases}
\end{aligned}
$$

Here $\left(\frac{z}{p}\right)$ is the Legendre symbol and

$$
g_{1}=\sum_{a=0}^{p-1} v^{a^{2}}=\left(v-v^{-1}\right)^{(p-1) / 2} \prod_{k=1}^{(p-1) / 2}[2 k-1]
$$

is the Gauss sum. In particular,

$$
\begin{aligned}
X & =\left\{U_{0}\right\}=-\frac{p}{\left(v-v^{-1}\right)^{2}}=\frac{(-1)^{(p+1) / 2}}{\left(v-v^{-1}\right)^{2}} g_{1}^{2}, \\
C_{ \pm} & =\left\{U_{ \pm 1}\right\}= \pm\left(\frac{\mp \overline{2}}{p}\right) \frac{g_{1} v^{ \pm 3 / 2}}{v-v^{-1}} .
\end{aligned}
$$

Let $\varphi_{p}: \mathcal{K}_{p} \rightarrow \mathbb{Z} / p$ denote the ring homomorphism such that $\varphi_{p}(v)=1$. Then for any category of $\operatorname{so}(3)$ type, $\varphi_{p}\left(\theta_{a}\right)=1$ and $\varphi_{p}(X)=0$. Hence according to Corollary $3.14, \mathcal{Z}_{\mathcal{K}_{p}}^{4} \otimes \varphi_{p}=\mathcal{Z}_{\mathbb{Z} / p}^{2}$ is an invariant of 2-complexes which vanishes on $S^{2}$. But at the same time, $\mathcal{Z}_{\mathbb{Z} / p}^{2}$ is a reduction of a modular invariant and therefore Theorem 2.11 implies that in Euler characteristic $\geq 1$ it depends only on homology in the following way:

4.2. Proposition. Suppose that $P$ is a 2-complex with Euler characteristic greater than or equal to 1 and $H_{1}(P)=\left(\bigoplus_{i} \mathbb{Z} / t_{i}\right) \oplus \mathbb{Z}^{b_{1}}$ and $H_{2}(P)$ $=\mathbb{Z}^{b_{2}}$. Then $\mathcal{Z}_{\mathbb{Z} / p}^{2}(P)$ is 0 if $b_{2}>0$ or if $p$ divides $t_{i}$ for some $i$, and it is $\prod_{i} \bar{t}_{i}^{2} \in \mathbb{Z} / p \mathbb{Z}$ otherwise.

Proof. We only need to observe that if $n$ is prime to $p$ and if $\operatorname{sgn}(n)$ denotes the sign of $n$ then

$$
\varphi_{p}\left(Y_{n}\right)=\varphi_{p}\left(\frac{\left\{U_{n}\right\}}{C_{\mathrm{sgn}(n)}}\right)=\left(\frac{n}{p}\right) \bar{n} .
$$

Otherwise $\varphi_{p}\left(Y_{n}\right)=0$.

4.3. The above statement is no longer true in the case when the Euler characteristic of the complex is smaller than or equal to 0 . The simplest counterexample comes from comparing the invariant of $S^{2} \vee \bigvee^{2} S^{1}$ (which is 0 ) and the one of the 2-complex $C$ with $\widehat{C}=\left\langle x, y \mid x y x^{-1} y^{-1}\right\rangle$. To evaluate $\mathcal{Z}_{\mathbb{Z} / p}^{2}(C)$ we take the 4 -thickening $(M, C)$ presented by the leftmost diagram 
in Figure 9. Together with 3.4 Figure 9 shows that

$$
\mathcal{Z}_{\mathcal{K}_{p}}^{4}(M)=\sum_{a=0}^{l} 1=l+1 .
$$

Hence $\mathcal{Z}_{\mathbb{Z} / p}^{2}(C)=\varphi_{p}\left(\mathcal{Z}_{\mathcal{K}_{p}}^{4}(M)\right)=(l+1) \bmod p$. In particular, for the explicit example of an so(3) type category presented in 5.1 with $p=5$ and $l=1$, we have $\mathcal{Z}_{\mathbb{Z} / p}^{2}(C)=2 \neq 0$.

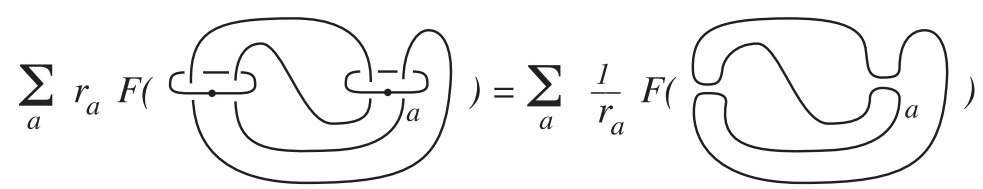

Fig. 9. Evaluation of $\mathcal{Z}_{\mathbb{Z} / p}^{2}(C)$ with $\widehat{C}=\left\langle x, y \mid x y x^{-1} y^{-1}\right\rangle$

4.4. Bounding properties and cyclotomic orders of 3-manifolds. Let $h=$ $v-1 \in \mathcal{K}_{p}$. We refer the reader to [5] for the definition and properties of the $p$-order $o_{p}$ of an element in $\mathcal{K}_{p}^{\prime}$, indicating just that for $x, y \in K_{p}, o_{p}(x)$ is the exponent of the highest power of $h$ which divides $x$ (as an element in $\left.K_{p}\right)$ and $o_{p}(x / y)=o_{p}(x)-o_{p}(y)$.

Let $\mathcal{C}$ be an so(3) type category over $\mathcal{K}_{p}$ and let $W$ be a 3 -manifold obtained by surgery on a link $L$. The level $p$ quantum so(3) invariant of $W$, as defined in [5], is given by

$$
\tau_{p}(W)=\{L\} /|L| \quad \text { where }|L|=C_{+}^{\sigma_{+}} C_{-}^{\sigma_{-}}\left(\frac{X}{h^{l}}\right)^{\sigma_{0}},
$$

and $o_{p}\left(\tau_{p}(W)\right)$ is called the cyclotomic order of $W$. Moreover, according to Theorem 4.2 in [5], $\tau_{p}$ takes values in $\mathcal{K}_{p}$. Let now $M$ be a 4 -thickening represented by a K-link $\dot{L}$ with $n$ dotted components and let $L$ denote the link obtained from $\dot{L}$ by removing all the dots. Since an so(3) type category is modular,

$$
\mathcal{Z}_{\mathcal{K}_{p}}(M)=\frac{\{L\}}{X^{n}}=N_{p} \tau_{p}(\partial M)
$$

where

$$
\begin{aligned}
N_{p} & =\left(\frac{C_{+}}{C_{-}}\right)^{\operatorname{index}(M) / 2}\left(\frac{X}{h^{p-3}}\right)^{\sigma_{0} / 2} X^{(\chi(M)-1) / 2} \\
& =(-1)^{\left(\sigma_{+}+\sigma_{0}-n\right)(p+1) / 2}\left(\frac{g_{1}}{v-v^{-1}}\right)^{\chi(M)-1}\left(1+v^{-1}\right)^{\sigma_{0}(p-3) / 2} .
\end{aligned}
$$

Since $o_{p}\left(g_{1} /\left(v-v^{-1}\right)\right)=(p-3) / 2$, it follows that

$$
o_{p}\left(\tau_{p}(\partial M)\right)+(\chi(M)-1) \frac{p-3}{2}=o_{p}\left(\mathcal{Z}_{\mathcal{K}_{p}}^{4}(M)\right) \geq 0 .
$$


So, we can give the following lower bound for the cyclotomic order of a 3-manifold:

4.5. Proposition. If a 3-manifold $W$ bounds a connected 4-dimensional manifold 2-handlebody $M$, then $o_{p}\left(\tau_{p}(\partial M)\right) \geq(1-\chi(M))(p-3) / 2$.

In any case this is a stronger statement than the bound for $o_{p}\left(\tau_{p}(\partial M)\right)$ given in [5]. It may be interesting to look at it as a lower bound for the Euler characteristic of the set of 4-thickenings with fixed boundary:

$$
\min _{M: \partial M=W} \chi(M) \geq 1-\frac{2}{p-3} o_{p}\left(\tau_{p}(W)\right),
$$

even if this bound is not sharp as can be seen from the example $W=L(n, 1)$.

5. Generating finite semisimple categories over a ring. The existence of such categories is a numerical and not theoretical result of the project in [23] where the category data can be viewed and downloaded. Here we just outline how such categories have been generated. For the sake of this work it is probably enough to present explicitly the smallest example as is done in Section 5.1, but we think it is important to indicate that the existence of a $\mathbb{Z} / p$-reduction of finite semisimple tortile categories coming from representations of quantum groups is quite a general phenomenon.

Let $\mathbf{g}$ be a Lie algebra and $p$ be greater than the Coxeter number of $\mathbf{g}$. To any such data in [6] there is associated a finite semisimple monoidal category $\overline{\mathcal{C}}_{p}$ (see [19] for the $\mathrm{sl}(2)$ case) which is a quotient of a subcategory of the category of representations of the quantum enveloping algebra of $\mathbf{g}$ over the field $\mathcal{K}_{p}^{\prime}$. The set $S$ of simple objects of $\overline{\mathcal{C}}_{p}$ correspond to the simple representations whose highest weights are contained in the open standard alcove of $\mathbf{g}$. The tortile structure of $\overline{\mathcal{C}}_{p}$ is explained in Chapter 6.1 and 32 in [14]. Moreover, for any $a \in S, \theta_{a}=v^{t(a)}$ for some $t(a) \in \mathbb{Z} / p\left({ }^{6}\right)$.

The algorithms in [23] generate numerically the tortile subcategory $\overline{\mathcal{C}}_{p}^{0}$ of $\overline{\mathcal{C}}_{p}$ whose simple objects are the representations in $S$ with highest weights in the root lattice of $\mathbf{g}$. These algorithms consist of the following main steps:

(i) explicit evaluation of the algebra generators at the representations in $S^{0}$ in the Lakshmibai-Sishadri bases;

(ii) explicit evaluation of the set of bases for the spaces $\operatorname{hom}_{\overline{\mathcal{C}}_{p}^{0}}(a, b c)$ and their duals;

(iii) computing the block matrices representing the commutativity and associativity morphisms with respect to the bases in (ii).

$\left({ }^{6}\right)$ The quantum Casimir operator (the twist), as described explicitly [13, 6.1.7], acts on any presentation $a \in S$ with highest weight $\lambda$ as multiplication by $v^{s(\lambda) / d}$, where $s$ is an integer-valued function on the weight space of $\mathbf{g}$ and $d$ is the determinant of the Cartan matrix of $\mathbf{g}$ and it is therefore prime to $p$ and can be inverted in $\mathbb{Z} / p$. 
The categories generated so far are the following:

\begin{tabular}{lcccc}
\hline algebra & A1 & A2 & C2 & G2 \\
\hline prime & $5,7,11,13,17$ & 7,11 & 7,11 & 11,13 \\
\hline
\end{tabular}

Except G2, $p=13$, all of the categories listed above live over the ring $\mathcal{K}_{p}$, i.e. with respect to the chosen bases of the spaces $\operatorname{hom}_{\overline{\mathcal{C}}_{p}^{0}}(a, b \otimes c)$ the forms, coforms, associativity and commutativity morphisms and their inverses are represented by matrices with $\mathcal{K}_{p}$-coefficients. Moreover, all of these categories are modular with $X=\sum_{a \in S} r_{a}^{2}$ and $\varphi_{p}(X)=0$. In particular, any of them leads to a modular invariant $\mathcal{Z}_{\mathcal{K}_{p}}^{4}$ of 4 -thickenings and, according to 3.14 , except $\mathrm{G} 2, p=13$, all these invariants have a $\mathbb{Z} / p$-reduction.

5.1. The category $\overline{\mathcal{C}}_{5}^{0}$ for $\mathbf{g}=\operatorname{sl}(2)$. We present here the smallest possible example corresponding to $\mathrm{g}=\operatorname{sl}(2), p=5$. There are only two simple objects, corresponding to the trivial representation $\mathbf{1}$ and the representation $a$ of highest weight $2: S^{0}=\{\mathbf{1}, a\}$. In $\overline{\mathcal{C}}_{5}^{0}$,

$$
a \diamond a \simeq 1 \oplus a .
$$

The category is equivalent to one with objects $A(n, m)=\mathcal{K}_{p}^{n} \oplus \mathcal{K}_{p}^{m}$, where $n$ and $m$ correspond to the number of trivial summands and the number of $a$ summands in $A$. The morphisms between two objects $A(n, m) \rightarrow A(k, l)$ are given by block matrices in $\mathcal{K}_{p}^{n \times k} \oplus \mathcal{K}_{p}^{m \times l}$ acting on the left. The tortile structure is defined by the product $A(n, m) \diamond A(k, l)=A(n k+m l, n l+m l$ $+m k)$ and the following morphisms:

$$
\begin{aligned}
& \gamma_{a, a}=\left(\begin{array}{cc}
1 & 0 \\
0 & -v^{2}
\end{array}\right), \quad \gamma_{a, a}^{-1}=\left(\begin{array}{cc}
1 & 0 \\
0 & -v^{3}
\end{array}\right) \in \mathcal{K}_{p} \oplus \mathcal{K}_{p} ; \\
& \alpha_{a, a, a}=\alpha_{a, a, a}^{-1}=\left(\begin{array}{ccc}
1 & 0 & 0 \\
0 & v^{2}+v^{3} & 1+v+v^{3} \\
0 & v+2 v^{2}+v^{3} & -v^{2}-v^{3}
\end{array}\right) \in \mathcal{K}_{p} \oplus \mathcal{K}_{p}^{2 \times 2} \\
& \Lambda_{a}=\left(\begin{array}{l}
1 \\
0
\end{array}\right) \in \mathcal{K}_{p} \oplus \mathcal{K}_{p} ; \\
& \lambda_{a}=\left(\begin{array}{ll}
r_{a} & 0
\end{array}\right) \in \mathcal{K}_{p} \oplus \mathcal{K}_{p}, \quad r_{a}=1+v+v^{3} ; \\
& \theta_{a}=v, \quad X=3+v+v^{3}, \quad C_{+}=-v^{2}+v, \quad C_{-}=-v^{3}+v^{4} .
\end{aligned}
$$

The $\mathbb{Z} / p$-reduction of this category gives exactly the symmetric category described in 10.7 of [18].

Finally, we observe that the HKR-type invariants of 2-complexes, defined in [4] from unimodular triangular Hopf algebras, in many cases are also 
reductions. This usually happens because the triangular algebra itself is obtained by a change of ring from a factorizable quasitriangular algebra (the factorizability condition leads to modular HKR-type invariants of 4thickenings). In conclusion, it seems like the search for interesting invariants of 2-complexes should start by looking at possible reductions of non-modular invariants of 4 -thickenings constructed either from premodular categories (see [2] for examples), or from non-factorizable unimodular quasitriangular Hopf algebras.

\section{References}

[1] J. Andrews and M. Curtis, Free groups and handlebodies, Proc. Amer. Math. Soc. 16 (1965), 192-195.

[2] A. Beliakova and C. Blanchet, Modular categories of types B, C and D, Comment. Math. Helv. 3 (2001), 467-500.

[3] I. Bobtcheva, On Quinn's invariants of 2-dimensional CW complexes, in: Contemp. Math. 233, Amer. Math. Soc., 1999, 69-95.

[4] I. Bobtcheva and M. G. Messia, HKR-type invariants of 4-thickenings of 2-dimensional $C W$-complexes, arXiv:math.QA/0206307.

[5] T. Cochran and P. Melvin, Quantum cyclotomic orders of 3-manifolds, Topology 40 (2001), 95-125.

[6] S. Gelfand and D. Kazhdan, Examples of tensor categories, Invent. Math. 109 (1992), 595-617.

[7] R. E. Gompf and A. I. Stipsicz, 4-Manifolds and Kirby Calculus, Grad. Stud. Math. 20, Amer. Math. Soc., Providence, RI, 1999.

[8] C. Hog-Angeloni, W. Metzler and A. Sieradski (eds.), Two-Dimensional Homotopy and Combinatorial Group Theory, London Math. Soc. Lecture Note Ser. 197, Cambridge Univ. Press, 1993.

[9] G. Huck, Embeddings of acyclic 2-complexes in $S^{4}$ with contractible complement, in: Lecture Notes in Math. 1440, Springer 1990, 122-129.

[10] T. Kerler, Bridged links and tangle presentations of cobordism categories, Adv. Math. 141 (1999), 207-281.

[11] -, Genealogy of nonperturbative quantum invariants of 3-manifolds: the surgical family, in: Geometry and Physics (Aarhus, 1995), Lecture Notes in Pure Appl. Math. 184, Dekker, New York, 1997, 503-547.

[12] R. Kirby, The Topology of 4-Manifolds, Lecture Notes in Math. 1374, Springer, 1980.

[13] R. Kirby and P. Melvin, The 3-manifold invariants of Witten and ReshetikhinTuraev for $\operatorname{sl}(2, \mathbb{C})$, Invent. Math. 105 (1991), 473-545.

[14] G. Lusztig, Introduction to Quantum Groups, Birkhäuser, Boston, 1993.

[15] S. MacLane, Categories for the Working Mathematician, Grad. Texts in Math. 5, Springer, 1971.

[16] K. Mueller, Probleme des einfachen Homotopietyps in niederen Dimensionen und ihre Behandlung mit Hilfsmitteln der topologischen Quantenfeldtheorie, dissertation, Johann-Wolfgang-Goethe-Univ., 2000.

[17] F. Quinn, Dual decompositions of 4-manifolds, Trans. Amer. Math. Soc. 354 (2002), 1373-1392. 
[18] F. Quinn, Lectures on axiomatic topological quantum field theory, in: LAS/Park City Math. Ser. 1, Amer. Math. Soc., 1995, 323-453.

[19] N. Yu. Reshetikhin and V. G. Turaev, Invariants of 3-manifolds via link polynomials and quantum groups, Invent. Math. 103 (1991), 547-597.

[20] M. C. Shum, Tortile tensor categories, J. Pure Appl. Algebra 93 (1994), 57-110.

[21] V. Turaev, Quantum Invariants of Knots and 3-Manifolds, de Gruyter Stud. Math. 18, de Gruyter, 1994.

[22] D. Yetter, State-sum invariants of 3-manifolds associated to artinian semisimple tortile categories, Topology Appl. 58 (1994), 47-80.

[23] http://www.math.vt.edu/quantum_topology.

Dipartimento di Scienze Matematiche

Università di Ancona

Via Brecce Bianche 1

60131 Ancona, Italy

E-mail: bobtchev@dipmat.univpm.it
Department of Mathematics Virginia Polytechnic Institute and State University Blacksburg, VA 24060, U.S.A. E-mail: quinn@calvin.math.vt.edu

Received 30 March 2004;

in revised form 19 July 2005 